\title{
ON THE EXPONENTIAL STABILITY OF LINEAR VISCOELASTICITY AND THERMOVISCOELASTICITY
}

\author{
BY
}

ZHUANGYI LIU (Department of Mathematics and Statistics, University of Minnesota, Duluth)

AND

SONGMU ZHENG (Institute of Mathematics, Fudan University, Shanghai 200433, China)

Abstract. The exponential stability of the semigroup associated with one-dimensional linear viscoelastic and thermoviscoelastic equations with several types of boundary conditions is proved for a class of kernel functions, including the weakly singular kernels.

1. Introduction. We consider the following integro-partial differential equations:

$$
\begin{aligned}
u_{t t}(x, t) & =\alpha u_{x x}(x, t)-\int_{0}^{\infty} g(s) u_{x x}(x, t-s) d s-\gamma c \theta_{x}(x, t), \\
\theta_{t}(x, t) & =-\gamma u_{x t}(x, t)+\theta_{x x}(x, t)
\end{aligned}
$$

with the initial conditions

$$
\begin{array}{rlrl}
u(x, 0)=u_{0}(x), \quad u_{t}(x, 0) & =u_{1}(x), & & \theta(x, 0)=\theta_{0}(x), \\
u(x, 0)-u(x,-s) & =w_{0}(x, s), \quad s \geq 0
\end{array}
$$

where $u_{0} \in H_{0}^{1}(\Omega), v_{0} \in L^{2}(\Omega), \theta_{0} \in L^{2}(\Omega), w_{0} \in L^{2}\left(\mathbf{R}^{+} ; g ; H_{0}^{1}(\Omega)\right), \Omega:=$ $(0, \pi)$, and $g(s)$ is a function satisfying the following conditions:

(g1) $g: \mathbf{R}^{+} \rightarrow \mathbf{R}, g \in C^{1}(0, \infty) \cap L^{1}(0, \infty)$,

(g2) $g \geq 0, g^{\prime}(s) \leq 0$ for $s \in \mathbf{R}^{+}$,

(g3) $\varepsilon=\alpha-\int_{0}^{\infty} g(s) d s>0$.

We denote by $H^{m}(\Omega)$ the Sobolov space consisting of all functions for which up to $m$ th-order generalized derivatives are all in $L^{2}(\Omega)$, and $H_{0}^{1}(\Omega)=\left\{f \in H^{1}(\Omega): f(0)\right.$ $=f(\pi)=0\}$. The "history space" $L^{2}\left(\mathbf{R}^{+} ; g ; H_{0}^{1}(\Omega)\right)$ consists of the $H_{0}^{1}(\Omega)$-valued functions $w$ on $\mathbf{R}^{+}:=[0, \infty)$ for which

$$
\|w\|_{L^{2}\left(\mathbf{R}^{+} ; g ; H_{0}^{1}(\Omega)\right)}^{2}:=\int_{0}^{\infty} g(s)\|w\|_{H_{0}^{1}(\Omega)}^{2} d s<\infty .
$$

Received April 22, 1993.

1991 Mathematics Subject Classification. Primary 35B40, 73H10, 73B30.

Key words and phrases. Linear viscoelasticity and thermoviscoelasticity, exponential stability, semigroup.

(c) 1996 Brown University 
We first consider the Dirichlet-Dirichlet boundary conditions

$$
u(i, t)=\theta(i, t)=0 \text { for } i=0, \pi .
$$

The set of equations (1.1)-(1.2) is a model for torsional motion of a linear viscoelastic rod of the Boltzmann type with thermal damping. The rod is assumed to have uniform mass density and length $\pi$. Here $u(x, t), \theta(x, t)$ represents angular displacement from equilibrium and temperature difference to the reference temperature at position $x$ and time $t$, respectively. $\gamma \geq 0, c>0$ are the thermo-mechanical coupling coefficients, $\alpha>0$ is the instantaneous elastic modulus, $\alpha-\int_{0}^{\infty} g(s) d s$ is the equilibrium elastic modulus. We refer the readers to Navaro's paper [Na] for a detailed discussion and derivation of the general form of 3-D linear thermoviscoelastic equations. The equations considered here are a simple 1-D version of Navaro's equations when the dependence of Helmholtz free energy on temperature difference history is neglected. Note that our $g(s)$ is his $-g^{\prime}(s)$.

Navaro proved the asymptotic stability for the 3-D linear thermoviscoelastic equations. When applied to Eqs. (1.1)-(1.2), his results imply that the solution $(u, \theta)$ is asymptotically stable in the space $H_{0}^{1}(\Omega) \times L^{2}(\Omega)$ if the kernel $g(s)$ satisfies conditions $(\mathrm{g} 2),(\mathrm{g} 3)$, and

$\left(\mathrm{g}^{\prime}\right) \quad g \in C^{1}[0, \infty), g, g^{\prime} \in L^{1}(0, \infty)$.

In 1989, Liu [L] extended Navaro's result for the weaker conditions (g1)-(g3), which allow $g(s)$ to have a singularity at $s=0$. After introducing the new variables

$$
v(x, t)=u_{t}(x, t), \quad w(x, t, s)=u(x, t)-u(x, t-s),
$$

and setting $z=(u, v, \theta, w)^{\mathrm{T}}$, Eqs. (1.1)-(1.2) can be written as an evolution equation

$$
\dot{z}(t)=\mathscr{A} z(t)
$$

on the Hilbert space $H=H_{0}^{1}(\Omega) \times L^{2}(\Omega) \times L^{2}(\Omega) \times L^{2}\left(\mathbf{R}^{+} ; g ; H_{0}^{1}(\Omega)\right):=U \times V \times$ $\Theta \times W$, with the norm induced by the inner product

$$
\begin{aligned}
\langle z, \bar{z}\rangle_{I I} & =\langle u, \bar{u}\rangle_{L^{\prime}}+\langle v, \bar{v}\rangle_{l^{\prime}}+\langle\theta, \bar{\theta}\rangle_{\Theta}+\langle w, \bar{w}\rangle_{w} \\
& =\varepsilon\left\langle u_{x}, \bar{u}_{x}\right\rangle+\langle v, \bar{v}\rangle+c\langle\theta, \bar{\theta}\rangle+\int_{0}^{\infty} g(s)\left\langle w_{x}, \bar{w}_{x}\right\rangle d s,
\end{aligned}
$$

so that the energy of $(1.1)-(1.2)$ is given by

$$
E(t)=\frac{1}{2}\left(\varepsilon\left\|u_{x}\right\|^{2}+\left\|u_{t}\right\|^{2}+c\|\theta\|^{2}+\int_{0}^{\infty} g(s)\left\|w_{x}\right\|^{2} d s\right) .
$$

Hereafter, $\langle\cdot, \cdot\rangle$ denotes the $L^{2}$ inner product, and $\|\cdot\|$ denotes the $L^{2}$ norm. The operator $\mathscr{A}$ is defined as

$$
\mathscr{A}\left(\begin{array}{c}
u \\
v \\
\theta \\
w
\end{array}\right)=\left(\begin{array}{c}
v \\
\varepsilon u_{x x}+\int_{0}^{\infty} g(s) w_{x x} d s-\gamma c \theta_{x} \\
-\gamma v_{x}+\theta_{x x} \\
v-w_{s}
\end{array}\right)
$$


with

$$
\mathscr{D}(\mathscr{A})=\left\{z \in H \mid \begin{array}{c}
\varepsilon u+\int_{0}^{\infty} g(s) w(s) d s, \theta \in H^{2}(\Omega) \cap H_{0}^{1}(\Omega), v \in H_{0}^{1}(\Omega) \\
w(s) \in H^{1}\left(\mathbf{R}^{+} ; g ; H_{0}^{1}(\Omega)\right), w(0)=0
\end{array}\right\} .
$$

If we expect to achieve exponential decay of the energy, we must assume that $g(s)$ decays exponentially as $s \rightarrow \infty$; so we also require that

(g4) $-g^{\prime}(s) \geq \delta g(s)$ for $s>0$ and some constant $\delta>0$.

REMARK 1.1. By Gronwell's inequality, condition (g4) implies that $g\left(\delta_{1}\right) e^{-\left(\delta-\delta_{1}\right) s}$ $\geq g(s)$ for $s>\delta_{1}>0$. If we pick $\delta_{1} \leq \delta$, then $g(s)$ decays exponentially for $s>\delta_{1}$. Note that (g4) allows $g(s)$ to have a singularity at $s=0$. The order of the singularity is less than 1 since $g(s)$ is a positive $L^{1}\left(\mathbf{R}^{+}\right)$function. For example, the weakly singular kernel of the form

$$
g(s)=c_{1} \frac{e^{-c_{3} s}}{s^{c_{2}}}, \quad 0<c_{2}<1, c_{1}, c_{3}>0
$$

satisfies ( $\mathrm{g} 4)$. This is a fractional derivative model modified by an exponential decay factor. Fractional derivative models have been used successfully to fit experimental complex modulus data for some real materials.

The main result in this paper is the following

TheOREM 1.1. Suppose that the kernel $g(s)$ satisfies conditions $(\mathrm{g} 1)-(\mathrm{g} 3)$. Then the operator $\mathscr{A}$ generates a $c_{0}$ semigroup $T(t)$ on the Hilbert space $H$. Moreover, if $g(s)$ also satisfies $(\mathrm{g} 4)$, then $T(t)$ is exponentially stable, i.e., there exist constants $M, \beta>0$ such that

$$
\|T(t)\|_{\mathscr{L}(H, H)} \leq M e^{-\beta t} \forall t>0
$$

The proof of this theorem will be given in Secs. 2 and 3. In Sec. 4, other types of boundary conditions will be discussed.

Concerning the exponential stability, to our knowledge, nothing was known in the literature for the linear thermoviscoelastic system. However, significant progress has been made recently on this issue for linear thermoelastic and linear viscoelastic systems, which are the special cases of system (1.1)-(1.2). If $g(s) \equiv 0,(1.1)-(1.2)$ become the thermoelastic equations

$$
\begin{gathered}
u_{t t}(x, t)=\alpha u_{x x}(x, t)-\gamma c \theta_{x}(x, t), \\
\theta_{t}(x, t)=-\gamma u_{x t}(x, t)+\theta_{x x}(x, t) .
\end{gathered}
$$

If $\gamma=0,(1.1)-(1.2)$ decouple to the linear viscoelastic equation

$$
u_{t \prime}(x, t)=\alpha u_{x x}(x, t)-\int_{0}^{\infty} g(s) u_{x x}(x, t-s) d s
$$

and the heat equation.

The thermoelastic energy of $(1.15)-(1.16)$ is defined as

$$
E(t)=\frac{1}{2}\left(\alpha\left\|u_{x}\right\|^{2}+\left\|u_{t}\right\|^{2}+c\|\theta\|^{2}\right) .
$$


In 1968, Dafermos in his pioneer work [Dal] proved that $E(t)$ converges to zero asymptotically if the initial state $\left(u_{0}, v_{0}, \theta_{0}\right)$ is in $H_{0}^{1}(\Omega) \times L^{2}(\Omega) \times L^{2}(\Omega)$ and $u, \theta$ satisfy Dirichlet-Dirichlet boundary conditions. Since then, much progress has been made to obtain the exponential decay rate of the energy for the system (1.15)-(1.16) with $\gamma>0$ :

$$
E(t) \leq M e^{-\beta t} E(0), \quad \forall t>0,
$$

In this direction, we refer to Slemrod [S], Hansen [H], Gibson et al [GRT] for the cases when $u$ and $\theta$ satisfy the Dirichlet-Neumann or Neumann-Dirichlet boundary conditions. We also refer to Rivera [R], Kim [K], Liu and Zheng [LZ] for the case of the Dirichlet-Dirichlet boundary condition. Notice that in $[\mathrm{S}]$ and $[\mathrm{R}]$, they required that the initial data were in $\mathscr{D}(\mathscr{A})$. However, this also leads to (1.19) using the argument by Kim in [K]. Recently, Burns and the authors in [BLZ] proved the exponential stability of the semigroup associated with system (1.15)-(1.16) subject to the boundary conditions which are all the possible combinations of the following type of boundary conditions at $x=0, \pi$ :
(i) $\left\{\begin{array}{l}u=0 \\ \theta=0\end{array}\right.$,
(ii) $\left\{\begin{array}{l}u=0 \\ \theta_{x}=0\end{array}\right.$,
(iii) $\left\{\begin{array}{l}\sigma=0 \\ \theta=0\end{array}\right.$,
(iv) $\left\{\begin{array}{l}\sigma=0 \\ \theta_{x}=0\end{array}\right.$

where $\sigma=u_{x}-\gamma \theta$ represents the stress. Thus the problem of the exponential stability of the system (1.15)-(1.16) has been completely solved.

The energy of the viscoelastic equation (1.17) is defined as

$$
E(t)=\frac{1}{2}\left(\varepsilon\left\|u_{x}\right\|^{2}+\left\|u_{t}\right\|^{2}+\int_{0}^{\infty} g(s)\left\|w_{x}\right\|^{2} d s\right) .
$$

In his paper [Da2], Dafermos proved that $E(t)$ tends to zero asymptotically when $u$ satisfies the Dirichlet boundary conditions and $u_{0} \in H_{0}^{1}(\Omega), u_{1} \in L^{2}(\Omega), w_{0} \in$ $L^{2}\left(\mathbf{R}^{+} ; g, H_{0}^{1}(\Omega)\right)$, and $g(s)$ satisfies the conditions $\left(\mathrm{gl}^{\prime}\right),(\mathrm{g} 2)-(\mathrm{g} 3)$, and is convex. Later on, a similar result without the assumption of convexity was obtained in [Da3]. The first result giving an explicit rate at which $E(t)$ decays to zero was proved by Day [D] in 1980. Let

$$
a(t)=\int_{t}^{\infty} g(s) d s+a(\infty)
$$

Assume $g(s)$ satisfies $(\mathrm{g} 1)-(\mathrm{g} 3)$, and $a(t)-a(\infty) \in L^{1}\left(\mathbf{R}^{+}\right)$and is log convex. Then $E(t)=o\left(t^{-1}\right)$ as $t \rightarrow \infty$ if the initial data $\left(u_{0}\right)_{x}$ and $\left(u_{1}\right)_{x}$ are uniformly bounded.

It was shown by Desch and Miller [DM1] that, in the case where the kernel $g(s)$ satisfies conditions $\left(\mathrm{gl}^{\prime}\right),(\mathrm{g} 2)-(\mathrm{g} 3)$, and decays exponentially, the solution $u$ and $u_{t}$ will also decay to zero exponentially at a rate no better than the kernel decays. Note that the initial history was taken to be zero. The assumption of a finite $g(0)$ is rather restrictive since different constitutive laws, such as singular kernels and fractional derivatives, have been proposed for viscoelastic solids.

As a consequence of Theorem 1.1, we have the following results concerning the exponential stability of the linear viscoelastic system (1.17).

Theorem 1.2. Suppose that the kernel $g(s)$ satisfies conditions $(\mathrm{g} 1)-(\mathrm{g} 3)$. Then the semigroup associated with system (1.17) is a $c_{0}$ semigroup on the Hilbert space 
$H=H_{0}^{1}(\Omega) \times L^{2}(\Omega) \times L^{2}\left(\mathbf{R}^{+} ; g ; H_{0}^{1}(\Omega)\right)$. Moreover, if $g(s)$ also satisfies $(\mathrm{g} 4)$, then the semigroup is exponentially stable, i.e., (1.19) holds for $E(t)$ defined by (1.18).

REMARK 1.2. Hannsgen and Wheeler [HW1, HW2] obtained the decay rate of the energy $E(t)$ for the viscoelastic system (1.17). When applied to the case of exponentially decaying kernels, their results also imply the exponential energy decay rate. However, they require additional smoothness of the kernel $g(s)$ and zero intial history. We also refer the readers to the work of Fabiano and Ito [FI1, FI2]. But their results do not cover the exponential stability for the singular kernels. After this paper was complete, we found a recent paper [FL] in which the authors obtained a similar result to Theorem 1.2 using a completely different method.

As we have seen, the thermal damping due to the heat conduction can induce exponential energy decay for the pure elastic system. However, it is a different story for the viscoelastic and thermoviscoelastic system. By the results in [DM1, DM2], we cannot expect the exponential decay rate for the thermoviscoelastic energy unless the kernel $g(s)$ already decays exponentially. On the other hand, our results suggest that when the viscoelastic energy decays exponentially, the corresponding thermoviscoelastic energy also decays exponentially. We still don't know whether the additional thermal damping will increase or decrease the stability margin.

The stabilizability of the viscoelastic system was investigated extensively in the late 1980s (for example, see [DM1, DM2, HRW, La, Le1, Le2]). It is known that the system is stabilizable only if the kernel $g(s)$ decays exponentially. The stabilization is better when $g(s)$ is singular at $s=0$. Furthermore, the abscissa of stability cannot be moved an arbitrary amount to the left by applying distributed control, [DM1, DM2]. When the system is subjected to dissipative boundary feedback, Lagnese [La] and Leugering [Le1, Le2] obtained estimates of the decay rate of the energy in simple linear viscoelastic systems (membranes, beams, and thin plates). They showed that the energy decays at a polynomial rate if $a(t)-a(\infty)$ satisfies a moment condition, and that it decays exponentially if the kernel decays exponentially. It is not clear whether the boundary feedback actually increases the margin of stability. In 1990, Hannsgen and Wheeler ([HW1], [HW2]) showed that in the case of zero initial history one may obtain the same rate of energy decay without the boundary feedback; thus, the decay is attributable to the internal viscoelastic damping.

2. Well-posedness. In this section we show the well-posedness of the evolution equation (1.8). To do this we argue that the operator $\mathscr{A}$ satisfies the hypotheses of a corollary of the Lumer-Phillips Theorem (see [Pa]) and hence generates a $C_{0}$ semigroup of contractions on $H$.

Lemma 2.1. $\mathscr{A}$ and its adjoint $\mathscr{A}^{*}$ are dissipative.

Proof. For every $z \in \mathscr{D}(\mathscr{A})$, we have

$$
\begin{aligned}
\langle\mathscr{A} z, z\rangle_{H}= & \langle v, u\rangle_{U^{U}}+\left\langle\varepsilon u_{x x}-\int_{0}^{\infty} g(s) w_{x x}(s) d s-\gamma c \theta_{x}, v\right\rangle_{V} \\
& +\left\langle-\gamma v_{x}+\theta_{x x}, \theta\right\rangle_{\Theta}+\left\langle v-w_{s}, w\right\rangle_{W} \\
= & -c\left\|\theta_{x}\right\|^{2}-\left\langle w_{s}, w\right\rangle_{W} .
\end{aligned}
$$


Note that $g(s)\left\|w_{x}(s)\right\|^{2}, g(s)\left\|w_{s x}\right\|^{2}$ are both in $L^{1}\left(\mathbf{R}^{+}\right)$since $z \in \mathscr{D}(\mathscr{A})$. Therefore,

$$
\lim _{s \rightarrow \infty} g(s)\left\|w_{x}(s)\right\|^{2}=0
$$

and $s g(s)\left\|w_{s . x}(s)\right\|^{2}$ is bounded near $s=0$. Furthermore,

$$
\lim _{s \rightarrow 0} g(s)\left\|w_{x}(s)\right\|^{2}=\lim _{s \rightarrow 0} s\left[s g(s)\left\|\frac{w_{x}(s)}{s}\right\|^{2}\right]=0 .
$$

It follows from integrating by parts and (2.2)-(2.3) that

$$
\left\langle w_{s}, w\right\rangle_{W}=-\frac{1}{2} \int_{0}^{\infty} g^{\prime}(s)\left\|w_{x}\right\|^{2} d s .
$$

Since $g(s)$ is nonincreasing, we obtain

$$
\langle\mathscr{A} z, z\rangle_{H}=-c\left\|\theta_{x}\right\|^{2}+\frac{1}{2} \int_{0}^{\infty} g^{\prime}(s)\left\|w_{x}\right\|^{2} d s \leq 0 .
$$

By a straightforward computation we obtain

$$
\mathscr{A}^{*}\left(\begin{array}{c}
u \\
v \\
\theta \\
w
\end{array}\right)=\left(\begin{array}{c}
-v \\
-\varepsilon u_{x x}-\int_{0}^{\infty} g(s) w_{x x} d s+\gamma c \theta_{x} \\
+\gamma v_{x}+\theta_{x x} \\
-v+\frac{g^{\prime}(s)}{g(s)} w+w_{s}
\end{array}\right),
$$

and

$$
\mathscr{D}\left(\mathscr{A}^{*}\right)=\left\{\begin{array}{l|l}
z \in H & \begin{array}{c}
\varepsilon u+\int_{0}^{\infty} g(s) w(s) d s, \theta \in H^{2}(\Omega) \cap H_{0}^{1}(\Omega), v \in H_{0}^{1}(\Omega) \\
w(s) \in H^{1}\left(\mathbf{R}^{+} ; g ; H_{0}^{1}(\Omega)\right), \frac{g^{\prime}(s)}{g(s)} w \in L^{2}\left(\mathbf{R}^{+} ; g ; H_{0}^{1}(\Omega)\right)
\end{array}
\end{array}\right\} .
$$

For any $z \in \mathscr{D}\left(\mathscr{A}^{*}\right)$, we have

$$
\begin{aligned}
\left\langle\mathscr{A}^{*}{ }_{z}, z\right\rangle_{H}=\langle- & v, u\rangle_{U^{\prime}}+\left\langle-\varepsilon u_{x x}-\int_{0}^{\infty} g(s) w_{x x}(s) d s+\gamma c \theta_{x}, v\right\rangle_{V} \\
& +\left\langle\gamma v_{x}+\theta_{x x}, \theta\right\rangle_{\Theta}+\left\langle-v+\frac{g^{\prime}(s)}{g(s)} w+w_{s}, w\right\rangle_{w^{\prime}} \\
= & -c\left\|\theta_{x}\right\|^{2}-\left\langle\frac{g^{\prime}(s)}{g(s)} w+w_{s}, w\right\rangle_{W} \\
= & -c\left\|\theta_{x}\right\|^{2}+\frac{1}{2} \int_{0}^{\infty} g^{\prime}(s)\left\|w_{x}\right\|^{2} d s-\lim _{s \rightarrow 0} \frac{1}{2} g(s)\left\|w_{x}(s)\right\|^{2} \leq 0 .
\end{aligned}
$$

Thus the dissipativeness of $\mathscr{A}$ and $\mathscr{A}^{*}$ is proved.

Lemma 2.2. $\mathscr{A}$ is closed.

Proof. Let $z_{n}=\left(u_{n}, v_{n}, \theta_{n}, w_{n}\right)^{\mathrm{T}} \in \mathscr{D}(\mathscr{A})$. Suppose

$$
z_{n} \rightarrow z=(u, v, \theta, w)^{\mathrm{T}}
$$


and

$$
\mathscr{A} z_{n} \rightarrow(\phi, \psi, \xi, h)^{\mathrm{T}}
$$

in $H$. Then

$$
\begin{aligned}
v_{n} \rightarrow \phi \quad & \text { in } H_{0}^{1}(\Omega), \\
\varepsilon\left(u_{n}\right)_{x x}+\int_{0}^{\infty} g(s)\left(w_{n}\right)_{x x} d s-\gamma c\left(\theta_{n}\right)_{x} \rightarrow \psi & \text { in } L^{2}(\Omega), \\
-\gamma\left(v_{n}\right)_{x}+\left(\theta_{n}\right)_{x x} \rightarrow \xi & \text { in } L^{2}(\Omega), \\
v_{n}-\left(w_{n}\right)_{s} \rightarrow h & \text { in } L^{2}\left(\mathbf{R}^{+}, g, H_{0}^{1}(\Omega)\right) .
\end{aligned}
$$

From (2.8) and (2.10) we have $v_{n} \rightarrow v$ in $H_{0}^{1}(\Omega)$ and $\phi=v$. Similarly, (2.8) and (2.13) yield $w_{n} \rightarrow w$ in $H^{1}\left(\mathbf{R}^{+}, g, H_{0}^{1}(\Omega)\right), w(0)=0$ and $h=v-w_{s}$. By (2.12) and the inequality $\left\|\theta_{x}\right\|^{2} \leq\|\theta\|^{2}\left\|\theta_{x x}\right\|^{2}$, we obtain $\theta_{n} \rightarrow \theta$ in $H_{0}^{1}(\Omega) \cap H^{2}(\Omega)$ and $\xi=-\gamma v_{x}+\theta_{x x}$. Lastly, by (2.8) and (2.11), we have $u_{n} \rightarrow u$ in $H_{0}^{1}(\Omega) \cap H^{2}(\Omega)$ and $\psi=\varepsilon u_{x x}+\int_{0}^{\infty} g(s) w_{x x} d s-\gamma c \theta_{x}$. Thus $z \in \mathscr{D}(\mathscr{A})$ and $\mathscr{A} z=(\phi, \psi, \xi, h)^{\mathrm{T}}$.

By Corollary 4.4 in [Pa], we have proved that $\mathscr{A}$ is the infinitesimal generator of a $C_{0}$-semigroup, $T(t)$, of contractions on the Hilbert space $H$.

3. Exponential stability. In this section, we will prove the exponential stability of the semigroup $T(t)$. Our proof relies on the following characteristic condition [Hu, Theorem $2^{\prime}$, p. 51] for an exponentially stable $C_{0}$-semigroup on Hilbert space $H$.

THeOREM 3.1. A $C_{0}$-semigroup $T(t)=e^{t .8}$ on Hilbert space $H$ is exponentially stable if and only if

$$
\sup \{\operatorname{Re} \lambda ; \lambda \in \sigma(\mathscr{A})\}<0
$$

and

$$
\sup _{\operatorname{Re} \lambda \geq 0}\left\|(\lambda-\mathscr{A})^{-1}\right\|=K<+\infty
$$

hold. Here $\sigma(\mathscr{A})$ stands for the spectrum of $\mathscr{A}$.

Proof of Theorem 1.1. We use the contradiction argument. Suppose the conclusion is not true. Then by Theorem 3.1 one of (3.1) and (3.2) must fail to hold.

Consider first the case when (3.2) fails to hold. Then there exist a sequence of $\lambda_{n} \in C$ and a sequence of $h_{n} \in \mathscr{D}(\mathscr{A}) \subset H$ with $\operatorname{Re} \lambda_{n} \geq 0$ and $\left\|h_{n}\right\|_{H}=1$ such that $($ as $n \rightarrow+\infty)$

$$
\lim _{n \rightarrow \infty}\left\|\left(\lambda_{n} I-\mathscr{A}\right) h_{n}\right\|_{H}=0
$$

which is equivalent to

$$
\begin{aligned}
\lambda_{n} h_{n}^{(1)}-h_{n}^{(2)} \rightarrow 0 & \text { in } U, \\
\lambda_{n} h_{n}^{(2)}-\varepsilon D^{2} h_{n}^{(1)}+\int_{0}^{\infty} g D^{2} h_{n}^{(4)} d s+\gamma c D h_{n}^{(3)} \rightarrow 0 & \text { in } V, \\
\lambda_{n} h_{n}^{(3)}+\gamma D h_{n}^{(2)}-D^{2} h_{n}^{(3)} \rightarrow 0 & \text { in } \Theta, \\
\lambda_{n} h_{n}^{(4)}-h_{n}^{(2)}+D_{s} h_{n}^{(4)} \rightarrow 0 & \text { in } W .
\end{aligned}
$$


It follows that

$$
\begin{aligned}
\operatorname{Re}\left\langle\left(\lambda_{n} I-\mathscr{A}\right) h_{n}, h_{n}\right\rangle_{H} & =\operatorname{Re} \lambda_{n}\left\|h_{n}\right\|_{H}^{2}-\operatorname{Re}\left\langle\mathscr{A} h_{n}, h_{n}\right\rangle_{H} \\
& =\operatorname{Re} \lambda_{n}+\left\|D_{x} h_{n}^{(3)}\right\|^{2}-\frac{1}{2} \int_{0}^{\infty} g^{\prime}(s)\left\|h_{n}^{(4)}\right\|_{L^{\prime}}^{2} d s \rightarrow 0 .
\end{aligned}
$$

Each term in (3.8) tends to zero since they are all nonnegative. By Poincarés inequality and condition (g4), we obtain

$$
\left\|h_{n}^{(3)}\right\|_{\Theta},\left\|h_{n}^{(4)}\right\|_{W} \rightarrow 0
$$

Thus

$$
\left\|h_{n}^{(1)}\right\|_{U}^{2}+\left\|h_{n}^{(2)}\right\|_{V^{\prime}}^{2} \rightarrow 1
$$

On the other hand, from (3.4) and Poincaré's inequality, we know that $\lambda_{n} h_{n}^{(1)}-h_{n}^{(2)}$ also converges to zero in $L^{2}$. Thus

$$
\lambda_{n}\left\langle h_{n}^{(1)}, h_{n}^{(2)}\right\rangle-\left\|h_{n}^{(2)}\right\|_{V}^{2} \rightarrow 0 \text {. }
$$

From (3.5) we have

$$
\lambda_{n}\left\langle h_{n}^{(2)}, h_{n}^{(1)}\right\rangle+\left\|h_{n}^{(1)}\right\|_{U}^{2}-\int_{0}^{\infty} g\left\langle D h_{n}^{(4)}, D h_{n}^{(1)}\right\rangle d s+\gamma c\left\langle D h_{n}^{(3)}, h_{n}^{(1)}\right\rangle \rightarrow 0 .
$$

It is easy to see that the last term in (3.12) converges to zero.

Since

$$
\begin{aligned}
\left|\int_{0}^{\infty} g\left\langle D h_{n}^{(4)}, D h_{n}^{(1)}\right\rangle d s\right| & \leq \int_{0}^{\infty} g\left\|D h_{n}^{(4)}\right\| \cdot\left\|D h_{n}^{(1)}\right\| d s \\
& \leq\left\|D h_{n}^{(1)}\right\| \cdot\left\|h_{n}^{(4)}\right\|_{W} \cdot \int_{0}^{\infty} g(s) d s \rightarrow 0,
\end{aligned}
$$

(3.12) becomes

$$
\lambda_{n}\left\langle h_{n}^{(2)}, h_{n}^{(1)}\right\rangle+\left\|h_{n}^{(1)}\right\|_{L^{\prime}}^{2} \rightarrow 0 .
$$

Adding the complex conjugate of (3.11) to (3.14) we get

$$
2 \operatorname{Re} \lambda_{n}\left\langle h_{n}^{(2)}, h_{n}^{(1)}\right\rangle+\left\|h_{n}^{(1)}\right\|_{U}^{2}-\left\|h_{n}^{(2)}\right\|_{V}^{2} \rightarrow 0 .
$$

Therefore, it follows from (3.10) and (3.15) and $\operatorname{Re} \lambda_{n} \rightarrow 0$ that

$$
\left\|h_{n}^{(1)}\right\|_{U}^{2} \rightarrow \frac{1}{2}, \quad\left\|h_{n}^{(2)}\right\|_{V}^{2} \rightarrow \frac{1}{2} \text {. }
$$

Now we claim $\left|\lambda_{n}\right| \geq \delta>0$ for all $n$. Otherwise, from (3.4), $h_{n}^{(2)} \rightarrow 0$ in $U$ (at least a subsequence), so does in $V$. A contradiction.

Dividing (3.4) by $\lambda_{n}$ and applying (3.16) yields

$$
\left\|\frac{h_{n}^{(2)}}{\lambda_{n}}\right\|_{l}^{2} \rightarrow \frac{1}{2}
$$

The rest of the proof is to show that (3.17) is a contradiction. We rewrite (3.7) as

$$
h_{n}^{(4)}-\frac{h_{n}^{(2)}}{\lambda_{n}}+\frac{1}{\lambda_{n}} D_{s} h_{n}^{(4)} \rightarrow 0 \text { in } W \text {. }
$$


Under the conditions imposed on $g(s)$, we claim that $s^{2} g^{\prime}(s) \in L^{1}\left(\mathbf{R}^{+}\right)$. This is because

$$
-\int_{0}^{\infty} s^{2} g^{\prime}(s) d s=2 \int_{0}^{\infty} s g(s) d s<\infty
$$

where we have used the facts that the order of the singularity of $g(s)$ at $s=0$ is less than one, and $g(s)$ decays exponentially for large $s$. By $(\mathrm{g} 4)$, we see that $s^{2} g(s)$ is also in $L^{1}\left(\mathbf{R}^{+}\right)$. Then we can easily check that $s h_{n}^{(2)} / \lambda_{n} \in W$. Now, taking the inner product of (3.18) with $s h_{n}^{(2)} / \lambda_{n}$ in $W$ and using (3.17) yields

$$
\left\langle h_{n}^{(4)}, \frac{s h_{n}^{(2)}}{\lambda_{n}}\right\rangle_{w}-\left\|\frac{D h_{n}^{(2)}}{\lambda_{n}}\right\|^{2} \int_{0}^{\infty} s g d s+\frac{1}{\lambda_{n}} \int_{0}^{\infty} s g\left\langle D_{s} h_{n}^{(4)}, \frac{h_{n}^{(2)}}{\lambda_{n}}\right\rangle_{H^{1}} d s \rightarrow 0 .
$$

It is clear from (3.9) and (3.17) that the first term in (3.20) converges to zero. To estimate the third term in (3.20), we integrate by parts to get

$$
\int_{0}^{\infty} s g\left\langle D_{s} h_{n}^{(4)}, \frac{h_{n}^{(2)}}{\lambda_{n}}\right\rangle_{H^{\prime}} d s=-\int_{0}^{\infty} s g^{\prime}\left\langle h_{n}^{(4)}, \frac{h_{n}^{(2)}}{\lambda_{n}}\right\rangle_{H^{\prime}} d s-\int_{0}^{\infty} g\left\langle h_{n}^{(4)}, \frac{h_{n}^{(2)}}{\lambda_{n}}\right\rangle_{H^{\prime}} d s,
$$

then estimate each term on the right-hand side of (3.21),

$$
\begin{aligned}
\left|\int_{0}^{\infty} s g^{\prime}\left\langle h_{n}^{(4)}, \frac{h_{n}^{(2)}}{\lambda_{n}}\right\rangle_{H^{\prime}} d s\right| \leq \int_{0}^{\infty}-s g^{\prime}\left\|D h_{n}^{(4)}\right\| \cdot\left\|\frac{D h_{n}^{(2)}}{\lambda_{n}}\right\| d s \\
\leq\left(\int_{0}^{\infty}-s^{2} g^{\prime} d s\right)^{1 / 2} \cdot\left(\int_{0}^{\infty}-g^{\prime}\left\|D h_{n}^{(4)}\right\|^{2} d s\right)^{1 / 2}\left\|\frac{D h_{n}^{(2)}}{\lambda_{n}}\right\| \rightarrow 0, \\
\left|\int_{0}^{\infty} g\left\langle h_{n}^{(4)}, \frac{h_{n}^{(2)}}{\lambda_{n}}\right\rangle_{H^{1}} d s\right| \leq \int_{0}^{\infty} g\left\|D h_{n}^{(4)}\right\| \cdot\left\|\frac{D h_{n}^{(2)}}{\lambda_{n}}\right\| d s \\
\leq\left(\int_{0}^{\infty} g\left\|D h_{n}^{(4)}\right\|^{2} d s\right)^{1 / 2} \cdot\left(\int_{0}^{\infty} g d s\right)^{1 / 2}\left\|\frac{D h_{n}^{(2)}}{\lambda_{n}}\right\| \rightarrow 0 .
\end{aligned}
$$

Thus the third term of (3.20) also converges to zero. Now the second term of (3.20) must converge to zero. This contradicts (3.17). Now we prove that (3.1) also holds. Because $T(t)$ is a $C_{0}$ semigroup of contractions, $\rho(\mathscr{A})$ contains the set $\{\lambda|\operatorname{Re} \lambda\rangle$ $0\}$. For any $\sigma \in\left[0, \frac{1}{2 K}\right]$ and $\omega \in \mathbf{R}$, we have

$$
(\sigma-i w-\mathscr{A})^{-1}=\left(\frac{1}{4 K}+i w-\mathscr{A}\right)^{-1}\left[I-\left(\sigma+\frac{1}{4 K}\right)\left(\frac{1}{4 K}+i w-\mathscr{A}\right)^{-1}\right]^{-1} \text {. }
$$

Thus,

$$
\sup \{\operatorname{Re} \lambda ; \lambda \in \sigma(\mathscr{A})\}<-\frac{1}{4 K} .
$$

The proof of the theorem is completed.

REMARK 3.1. Throughout the proof of Theorem 1.1, we only used the first and fourth row of the evolution equation (1.8) to get a contradiction. Therefore, it also 
works for the viscoelastic equation (1.17) by letting $\gamma=0$ and deleting the heat equation. Thus Theorem 1.2 is proved.

4. Other boundary conditions. In this section, we discuss the exponential energy decay of system (1.1)-(1.4) with other types of boundary conditions. The method used in the last section can be easily modified to handle these cases.

(1). We first consider the cases that both ends of the rod are rigidly clamped. Then, $u(0, t)=u(\pi, t)=0$. We have discussed the case when both ends are kept at constant temperature. If one end is insulated, i.e,. $\theta(0, t)=0, \theta_{x}(\pi, t)=0$, the proof of Theorem 3.1 can be carried on without changes except that the domain of $\mathscr{A}$ should be modified to fit the boundary conditions. However, if both ends of the rod are insulated, then the equilibrium of the temperature is a constant. Certain modifications of our proof are necessary. Note that, by integrating (1.2) with respect to $x$ from zero to $\pi$, and taking the boundary conditions of $u$ into account,

$$
\int_{0}^{\pi} \theta d x=\int_{0}^{\pi}\left(\theta+\gamma u_{x}\right) d x \int_{0}^{\pi}\left(\theta_{0}+\gamma\left(u_{0}\right)_{x}\right) d x \stackrel{\text { def }}{=} c_{1}
$$

for all $t$. Thus we let $\theta_{\text {new }}=\theta-c_{1} / \pi$. For simplicity of notation, we still denote $\theta_{\text {new }}$ by $\theta$ so that we again have system (1.1)-(1.4). The new state space is

$$
H=\left\{z \in U \times V \times \Theta \times W \mid \int_{0}^{\pi} \theta d x=0\right\},
$$

and the domain of $\mathscr{A}$ is modified according to the boundary conditions. By applying Poincaré's inequality to $\theta$ subject to the constraint $\int_{0}^{\pi} \theta d x=0$ and condition (g4), we still obtain (3.5). Then we can proceed as before to complete the proof.

(2). Secondly, suppose only one end (say, $x=0$ ) of the rod is rigidly clamped, and the other end is free. Then

$$
u(0, t)=\alpha u_{x}(\pi, t)-\int_{0}^{\infty} g(s) u_{x}(\pi, t-s) d s-\gamma c \theta(\pi, t)=0 .
$$

If $\theta(x, t)=0$ holds at least at one end of the rod, we will take the state space to be

$$
H=H_{L}(\Omega) \times L^{2}(\Omega) \times L^{2}(\Omega) \times L^{2}\left(\mathbf{R}^{+} ; g ; H_{L}(\Omega)\right)
$$

where $H_{L}(\Omega)=\left\{f \in H^{1}(\Omega): f(0)=0\right\}$. The domain of $\mathscr{A}$ should also be modified to fit the boundary conditions. Then carry on the proof as before. If both ends of the rod are insulated, we can again substitute the variable $\theta$ by $\theta-c_{1} / \pi$ as in part (1).

Acknowledgment. The second author was supported by NSFC 19071919, 19331040 and GEC 9024616.

\section{REFERENCES}

[BF] J. A. Burns and R. H. Fabiano, Feedback control of a hyperbolic partial differential equation with viscoelastic damping, Control Theory and Advanced Technology, Vol. 5, No. 2, 1989, pp. 157-188

[BLZ] J. A. Burns, Z. Liu, and S. Zheng, On the energy decay of a linear thermoelastic bar, J. Math. Anal. Appl. (to appear)

[D] W. A. Day, The decay of energy in a viscoelastic body, Mathematika, Vol. 27, 1980, pp. 268-286 
[Da1] C. M. Dafermos, On the existence and the asymptotic stability of solution to the equations of linear thermoelasticity, Arch. Rat. Mech. Anal. 29, 241-271 (1968)

[Da2] C. M. Dafermos, Asymptotic stability in viscoelasticity, Arch. Rat. Mech. Anal. 37, 297-308 (1970)

[Da3] C. M. Dafermos, An abstract Volterra equation with applications to linear viscoelasticity, J. Differential Equations 7, 554-569 (1970)

[DM1] W. Desch and R. K. Miller, Exponential stabilization of Volterra integrodifferential equations in Hilbert space, J. Differential Equations 70, 366-389 (1987)

[DM2] W. Desch and R. K. Miller, Exponential stabilization of Volterra integral equations with singular kernels, J. Integral Equations Appl. 1, 397-433 (1988)

[FI1] R. H. Fabiano and K. Ito, Semigroup theory and numerical approximation for equations in linear viscoelasticity, SIAM J. Math. Anal. 21 (2), 374-393 (1990)

[FI2] R. H. Fabiano and K. Ito, An approximation framework for equations in linear viscoelasticity with strongly singular kernels, Quart. Appl. Math. 52, 65-81 (1994)

[FL] M. Fabrizio and B. Lazzari, On the existence and asymptotic stability of solutions for linearly viscoelastic solids, Arch. Rat. Mech. Anal. 116, 139-152 (1991)

[GRT] J. S. Gibson, I. G. Rosen, and G. Tao, Approximation in control of thermoelastic systems, SIAM J. Control and Optimization 30 5, 1163-1189 (1992)

$[\mathrm{H}] \mathrm{S}$. W. Hansen, Exponential energy decay in a linear thermoelastic rod, J. Math. Anal. Appl. 167, 429-442 (1992)

[Hu] F. L. Huang, Characteristic condition for exponential stability of linear dynamical systems in Hilbert spaces, Ann. Differential Equations 1 (1), 43-56 (1985)

[HRW] K. B. Hannsgen, Y. Renardy, and R. L. Wheeler, Effectiveness and robustness with respect to time delays of boundary feedback stabilization in one-dimensional viscoelasticity, SIAM J. Control Optim. 26 5, 1200-1233 (1988)

[HWI] K. B. Hannsgen and R. L. Wheeler, Viscoelastic and boundary feedback damping: Precise energy decay rates when creep modes are dominant, J. Integral Equations Appl. 2, 495-527 (1990)

[HW2] K. B. Hannsgen and R. L. Wheeler, Moment conditions for a Volterra integral equation in a Banach space, Proceedings of Delay Differential Equations and Dynamical Systems, Claremont, Vol. 1475 of Springer Lecture Notes in Mathematics, Springer-Verlag, 1991, pp. 204-209

[J] S. Jiang, Global solution of the Neumann problem in one-dimensional nonlinear thermoelasticity, preprint, 1991

[K] J. U. Kim, On the energy decay of a linear thermoelastic bar and plate, SIAM J. Math. Anal. 23 (4), 889-899 (1992)

[L] Z. Liu, Approximation and control of a thermoviscoelastic system, Ph.D. dissertation, Virginia Polytechnic Institute and State University, Department of Mathematics, August, 1989

[La] J. Lagnese, Boundary Stabilization of Thin Plates, Vol. 10 of SIAM Studies in Applied Mathematics, Society for Industrial and Applied Mathematics, Philadelphia, 1989

[Le1] G. Leugering, On boundary feedback stabilization of a viscoelastic membrane, Dynamics and Stability of Systems 4 (1), 71-79 (1989)

[Le2] G. Leugering, On boundary feedback stabilizability of a viscoelastic beam, Proc. Roy. Soc. Edinburgh Sect. A 114 (1), 57-69 (1990)

[LZ] Z. Liu and S. Zheng, Exponential stability of the semigroup associated with a thermoelastic system, Quart. Appl. Math. 51, 535-545 (1993)

[Na] C. B. Navaro, Asymptotic stability in linear thermoviscoelasticity, J. Math. Appl. 65, 399-431 (1978)

[Pa] A. Pazy, Semigroups of Linear Operators and Applications to Partial Differential equations, Springer, New York, 1983

[R] J. E. M. Rivera, Energy decay rate in linear thermoelasticity, Funkcial Ekvac. 35, 19-30 (1992)

[S] M. Slemrod, Global existence, uniqueness, and asymptotic stability of classical smooth solutions in one-dimensional nonlinear thermoelasticity, Arch Rat. Mech. Anal. 76, 97-133 (1981)

[Sh] Y. Shibata, Neumann problem for one-dimensional nonlinear thermoelasticity, preprint, 1991 